\title{
Black Community Conversations About Opposing Ethnically Targeted Marketing of Unhealthy Foods and Beverages
}

\author{
Tiffany M. Eaton ${ }^{1} \cdot$ Shiriki Kumanyika ${ }^{1}$ (1) $\cdot$ Katherine Isselmann DiSantis ${ }^{2} \cdot$ Kenna Yadeta $^{3} \cdot$ Sonya Grier $^{4}$
}

Received: 8 June 2021 / Revised: 10 August 2021 / Accepted: 11 August 2021 / Published online: 20 August 2021

(c) W. Montague Cobb-NMA Health Institute 2021

\begin{abstract}
Efforts to reduce disparities in obesity prevalence affecting Black Americans are having limited success. One reason for this may be the disproportionate, ethnically targeted marketing of foods and beverages high in fat and sugar (FBHFS) to Black consumers. Such marketing promotes high consumption of FBHFS, leading to excess caloric intake and unintentional weight gain. We convened focus groups with Black men and women (total $n=57$ ) in collaboration with community groups in three localities to elicit their views, as consumers and parents/caregivers, about targeted FBHFS marketing and potential ways to combat it. At each location, trained community members facilitated two sets of focus groups: one for adults aged 18 to 25 years and another for adults aged 26 to 55 years who had a 3-to-17-year-old child at home. Each group met twice to discuss food and beverage marketing practices to Black communities and reviewed a booklet about ethnically targeted marketing tactics in between. A directed content analysis of participant comments identified and explored salient themes apparent from initial summarization of results. Results show how parents are concerned with and critical of pervasive FBHFS marketing. In particular, comments emphasize the involvement of Black celebrities in FBHFS marketing — how and why they engage in such marketing and whether this could be shifted towards healthier foods. These findings suggest a potential role for counter marketing efforts focused on Black celebrity endorsements of FBHFS, possibly with a youth focus. They also underscore the need for additional, qualitative exploration of Black consumer views of ethnically targeted FBHFS marketing more generally.
\end{abstract}

Keywords Childhood obesity $\cdot$ Celebrities $\cdot$ Food marketing $\cdot$ Black Americans $\cdot$ Health disparities

\section{Introduction}

The obesity epidemic is unrelenting and affects Black and Hispanic/Latino populations disproportionately [1]. In data from the U.S. National Health and Nutrition Examination Survey, obesity prevalence increased by $\sim$ sixteen percentage points in men and $\sim$ nine percentage points in women

Shiriki Kumanyika

Kumanyika@drexel.edu

1 Department of Community Health \& Prevention, Drexel University Dornsife School of Public Health, 3215 Market Street, 4th Floor, Philadelphia, PA 19104, USA

2 College of Population Health, Thomas Jefferson University, Philadelphia, PA 19107, USA

3 Intramural Training Program, National Institute of Drug Abuse, Biomedical Research Center, Baltimore, MD 21224, USA

4 Department of Marketing, Kogod School of Business, American University, Washington, DC 20016, USA between 1999-2000 and 2017-2018. During the same time period, obesity prevalence also increased in youth ages 6 years and older [1]. For example, prevalence in Black and Mexican American adolescents (ages 12 to 19) increased by seven and eight percentage points, respectively, compared to an increase of only two percentage points in White adolescents. By 2018, obesity prevalence was $50 \%$ in Black adults vs. $42 \%$ in Whites, $23 \%$ in Black children aged 6 to 11 years vs. $16 \%$ in White children in this age range, and $28 \%$ in Black adolescents vs. $16 \%$ in White adolescents $[1,2]$.

Food marketing practices contribute to consumption of foods that promote excess caloric intake and obesity, e.g., fast food, packaged snack foods, and sugary beverages [3]. Marketing of foods and beverages high in calories from fat and sugar (FBHFS) is viewed by nutrition experts as a major hindrance to the success of obesity prevention and control efforts [4-6]. Large manufacturers and restaurant chains invest billions of dollars to promote the purchase and consumption of FBHFS - far outspending public health or non-profit investments in marketing of foods that are less 
obesity-promoting [7-9]. A substantial proportion of this marketing is directed to children and adolescents, whodevelopmentally_are particularly susceptible to certain marketing tactics in traditional channels such as television, and particularly through heavy engagement with social media because of the uniquely engaging characteristics of digital media [6, 10-14]. Advertising susceptibility of older children and adolescents is related to identity formation, e.g., the influence of popular celebrities, and by social media interactions that facilitate self-expression and peer contact $[12,15]$.

Marketing of FBHFS to Black and Latino adults and children (referred to hereafter as "ethnically targeted marketing") is characteristic, and the strategies used result in greater exposure to FBHFS marketing compared to the population at large [16-19]. Ethnically targeted marketing takes many forms [10, 11, 20]. Higher exposure to FBHFS marketing results from a combination of the greater frequency of food advertisements on television programs viewed by Black people and higher media use [11, 21]. Moreover, strategies leverage Black identity, culture, and endorsements from Black entertainers or athletes-adding salience [22-24]. Advertisements and other promotions work in concert with tailored product design, placement of FBHFS products in accessible locations, and affordable pricing [16-18, 22, 25]. Black youth have "double or triple jeopardy" in that they are exposed to general audience child targeted marketing, to ethnically targeted child marketing, and to ethnically targeted marketing to Black adults or communities in general [23]. There is evidence that Black youth may also trust food advertising more than peers in other racial/ethnic minority groups [20]. These heavier FBHFS marketing exposures contribute to the higher obesity prevalence in Black communities and hinder related obesity prevention and control efforts.

Efforts to counter or regulate FBHFS marketing overall or marketing targeted by ethnicity to adults, youth, or both have had limited success $[5,7,26]$. Most have focused on youth because of their developmental vulnerability to marketing persuasion [6, 27]. Regulations that target food safety do not apply. Foods and beverages are essential for life and regulated as products presumed safe for human consumption, but "food safety" does not incorporate chronic disease risk. Moreover, food and beverage advertising is protected under the first Amendment to the U.S. Constitution [28, 29]. Current limitations on which foods can be marketed are based on relatively weak voluntary food and beverage industry agreements, applicable to companies who sign on to them and to children up to age 12 years, although adolescents are also susceptible [11, 13, 20, 30, 31]. Furthermore, companies are not accountable to adhere to these guidelines [32, 33].

Ethnically targeted marketing is a focus of research and advocacy within the public health nutrition and disparities research communities, but there is limited research on how Black adult consumers view or respond to such marketing to themselves or in relation to children in their care. Community voices on this issue are critical for informing effective solutions. We, therefore, engaged Black community members in conversations about this issue to explore their perspectives on marketing practices they might consider problematic and elicit their ideas for taking action to change these practices. We built on prior evidence indicating that Black community members may have relatively tolerant or even positive views of marketing and may like seeing themselves represented in ads while they may also express dislike for aspects that seem unfair, discriminatory, or harmful to Black communities [31, 34-36]. Greater insight into these divergent responses can support the development of interventions that can garner community support.

\section{Methods}

The design of this qualitative study called for a total of twelve focus groups in three geographic areas (four focus group sessions per site: two for younger adults, and two for adult caregivers) to obtain diverse perspectives from Black adults. The protocol, adapted from a two-part sensitization approach [35, 37], required convening each focus group about one week apart. The protocol was the same for each age group at each locality. Focus groups were moderated by trained facilitators from the local community, all Black adults.

\section{Study Settings}

Three local community-based organizations in Maryland (suburban), Mississippi (rural), and Ohio (urban) convened and conducted the focus groups with support from the research team. Each organization received modest compensation for their efforts. These organizations were well-known in their respective communities and had been previously involved with some type of food access program but had not previously engaged with food marketing issues as such. Leads from each site attended an orientation and planning meeting at which they learned about the study rationale, objectives, and proposed approach; provided feedback on the focus group protocol and informational booklet and discussed the logistics for implementation at their respective sites.

\section{Participant Recruitment}

Staff at each site served as contact persons and coordinated the advertisement, screening, and recruitment for the focus groups. The goal at each site was to recruit 10-15 men and 
women who self-identified as Black, who were either 26 to 55 years old and living in a household with a child aged 3-17 or were 18 to 25 years old and did not necessarily have children in their household. In addition, participants were considered eligible if they were willing to participate in two sessions of focus groups; were not employed by marketing firms or otherwise engaged in marketing research, advertising, public relations, or media industries; and lived in one of the geographical areas where the community partners were located. Each site lead recruited primarily through the distribution of study flyers sent directly to their respective clients or shared throughout their community and screened each participant to determine eligibility. Consent was obtained anonymously and remotely through a Qualtrics survey accessible only to the project director and project coordinator. Descriptive data collected remotely as part of the consent process obtained relevant demographic data to describe the participants at each site as a group. Participants were offered a $\$ 25$ gift card per session attended to offset out of pocket costs.

\section{Data Collection Protocol}

The focus group guide and study booklet were adapted from a sensitization protocol that was used in a previous study [35]. The present study materials were revised to update the content to include recent scenarios and examples and were piloted with each of the site leads to ensure they would be relevant to the three regions. Focus group scenarios and booklet content related to the "4 P's" of marketing [38]: (Product: 4 scenarios; Place: 5 scenarios; Promotion: 6 scenarios; Price: 4 scenarios; and one on marketing research). Focus Group Session 1 lasted for 90 minutes. Each scenario included an illustration and response question (Table 1). All focus groups were conducted in-person. Moderators presented ground rules and explained that they would show a series of scenarios on slides, ask for quick "yes," "no,"

Table 1 Scenario descriptions and questions posed from moderator guide for Session 1 focus groups

Session 1 Focus Groups*

Scenario described: [FAST FOOD COMPANY] is a lead sponsor at [POPULAR RAPPER]'s [MUSIC FESTIVAL] in [CITY]. In this picture,

TV personality, [POPULAR TV ACTOR], serves up fries from the [FAST FOOD COMPANY] truck

Question posed: Do you see this as marketing?

Scenario described: Supermarkets or large grocery stores are not easy to get to in every community

Question posed: Do you know of communities that don't have access to supermarkets or large grocery stores?

Scenario described: Smaller community stores tend to have higher prices than larger supermarkets or "big box" stores (e.g., Walmart) Question posed: Do you think this price difference is fair to consumers?

Scenario described: Detroit limited the proximity of fast food to schools to address health concerns related to childhood obesity

Question posed: Do you think public officials should use zoning laws to restrict what types of restaurants or food stores are available in a community?

Scenario described: Several states/cities have a specific sales tax on soft drinks and other sweet drinks. The idea is that taxing beverages will raise the price and consequently decrease consumption. Philadelphia enacted a beverage tax of $1.5 \%$ (e.g. about $2 \varnothing$ per $99 \not \subset$ drink) in 2017 . Studies have shown that Philadelphians now purchase fewer sugary drinks than comparison cities in New Jersey, which does not have a tax. But some community leaders oppose the tax, including some members of the city's Black Clergy

Question posed: Would you be in favor of a beverage tax in your state or city?

Scenario described: Research shows that Black consumers often add sugar to their tea when it is already sweetened. A [FAST FOOD COM-

PANY] decides to offer a sweeter version of tea at outlets that are in Black areas and promote regular tea in other areas

Question posed: Do you think people should be told that different products are being developed for them or promoted to them based on their race?

Scenario described: Some cigarette brands, cigarette flavors and cigarette packages have been designed to appeal especially to Black Americans, including Black youth

Question posed: Do you think companies should use people's racial identity to sell cigarettes/e-cigarettes?

Scenario described: Food and Beverage companies are using digital marketing to create highly personalized apps/websites that are attractive to youth

Question posed: Do you think this digital marketing helps companies sell their products?

Scenario described: Marketing agencies often use popular artist's songs to promote specific product brands for cars, clothes, beverages, etc Question posed: Do you think this type of ad influences what people buy?

Scenario described: A recent research report summarizes Black Americans' spending behaviors. A researcher from Nielsen, a global market research firm, stated "Our research shows that Black consumer choices have a 'cool factor' that has created a halo effect, influencing not just consumers of color but the mainstream as well."

Question posed: Do you feel good when you hear about this market research on Black consumers?

*Initial options for each question were: Yes/No/Unsure. The moderator used these responses to guide subsequent discussion. See text for explanation 
or "unsure" responses to the question posed, and then ask various group members to discuss their response, leading to a full group discussion.

The information booklet provided for each participant to take home after session 1 was entitled, "BIG BUSINESS: Food Marketing and the Health of Black Americans" (see Online Resource 1). The first two pages provided context for the scenarios within the booklet and explained the marketing concepts of the 4P's and targeting certain population segments, including Black adults and children, for the sale of certain products. The remaining twelve pages presented additional scenarios to elaborate on issues related to products (flavoring), food store availability (neighborhoods), beverage taxes (policy), and promotions (celebrities) with more specific explanations of how marketing strategies work.

Focus Group Session 2 lasted for 60 minutes and was a continuation of the discussion about the Session 1 scenarios and participants' review of the booklet. Moderators asked participants what stood out to them in the booklet and why. This session included other questions used to stimulate discussion and to ask about perceptions of needed change: "Has your opinion about any of the scenarios we showed previously changed after reading the booklet? How?," "What did you learn from the booklet that informs your opinion now?," and "In relation to foods and beverages what types of products would you like to see marketed in your communities? What would you like to see 'disappear'?".

\section{Community Meeting}

The research team provided each organization with a sitespecific summary of key findings organized in relevant categories such as healthier retail, healthier restaurants, and campaigns to raise awareness of targeted marketing. The feedback from each site was shared with a resource person with expertise in changing food and nutrition policies and practices in local communities to inform and facilitate next steps for actionable strategies. The team encouraged community collaborators to convene additional conversations about targeted marketing issues on their own, inviting a broad group of community stakeholders to discuss the study findings and determine how to use them locally, and to provide feedback from these sessions to the research team. When the conditions allowed, community meetings were to be audio recorded in order to include those perspectives in the qualitative analysis.

\section{Data Analysis}

Audio files were transcribed by a commercial vendor and analyzed as a single data set: data for sessions 1 and 2, age groups, and sites were combined. However, the source of text was noted to facilitate examination of consistency when identifying the emergence of themes: responses were checked to assess whether the theme was salient across age groups and sites. A directed analysis was used to identify participant views on celebrities' role in food and beverage marketing and their reflections and perspective on targeted marketing. This topic was identified through preliminary review and discussion of the overall findings with the objective of identifying potential focal points for action. Transcripts were coded using NVivo12 software. Analyses involved development of a preliminary codebook from analysis of a similar, prior study that included celebrity marketing content [36]. Additional emergent codes that identified views on food and beverage marketing, targeted marketing, and celebrities were also added to the codebook.

This deductive and inductive coding approach led to thematic development of salient themes that provided refined, extended, or divergent (new) ideas. The analysis was done by four of the authors, none of whom had attended or moderated the focus groups. Two authors (TME and KY) coded the transcripts. The codebook and the themes were reviewed, refined, and finalized based on discussions with two others (KD and SK). A reliability assessment was performed on all transcripts using NVivo to calculate the Kappa value for agreement and disagreement between coders. Complete agreement in coding correlates with a mean $\kappa$ of 1 , and a mean $\kappa$ of $0.81-0.99$ suggests strong agreement. Analysis of intercoder reliability for this study revealed a strong level of agreement (mean $\kappa=0.9$; range, 0.9-1.0) confirming the inter-coder reliability.

\section{Results}

\section{Participants}

Across sites, a total of 57 men and women participated in the focus group discussions for Session 1: 15 in Maryland, 22 in Mississippi, and 20 in Ohio. Out of the initial 57 participants, 45 returned for the Session 2 discussions: 13 in Maryland; 21 in Mississippi, and 11 in Ohio. Participant demographic data are shown in Table 2. These data include all 69 consented participants because non-participants could not be identified from the anonymous forms. As shown in Table 2, most were female (68\%), full- or part-time employed (87\%), and primary grocery shoppers for their households (75\%). Eighty-four percent reported eating out or ordering take-out at least 1 to 2 times a week; more than $80 \%$ watched TV on an average week or weekend day; and $93 \%$ used social media such as Twitter, Facebook, or Instagram (not shown in the table). Of the twelve possible sessions, eight recordings were obtained: four from session 1, three from session 2-and one from a community meeting held at one site. Recordings from five sessions were not usable due to technical problems. 
Table 2 Participant demographics $(n=69) *$

\begin{tabular}{lllll}
\hline Variable & $\begin{array}{l}\text { Maryland } \\
\text { participants } \\
n=15\end{array}$ & $\begin{array}{l}\text { Mississippi } \\
\text { participants } \\
n=23\end{array}$ & Ohio participants & Total \\
& & & & 69 \\
\hline Age & $6(40 \%)$ & $10(43 \%)$ & $13(42 \%)$ & $29(42 \%)$ \\
$\quad 18$ to 25 years & $9(60 \%)$ & $13(57 \%)$ & $18(58 \%)$ & $40(58 \%)$ \\
$\quad 26$ years and older & & & & \\
Gender & $9(60 \%)$ & $18(78 \%)$ & $19(63 \%)^{\dagger}$ & $46(68 \%)$ \\
$\quad \begin{array}{l}\text { Female } \\
\text { Male }\end{array}$ & $6(40 \%)$ & $5(22 \%)$ & $11(37 \%)^{\dagger}$ & $22(32 \%)$ \\
Education & $5(33 \%)$ & $14(61 \%)$ & $25(81 \%)$ & $44(64 \%)$ \\
$\quad$ Some college or less & $10(67 \%)$ & $9(39 \%)$ & $6(19 \%)$ & $25(36 \%)$ \\
$\quad$ College graduate or more & $9(60 \%)$ & $6(26 \%)$ & $11(35 \%)$ & $26(38 \%)$ \\
Married/living with partner & & & & \\
Employment status & $14(93 \%)$ & $21(91 \%)$ & $25(81 \%)$ & $60(87 \%)$ \\
$\quad \begin{array}{l}\text { Full-time or part-time } \\
\text { Retired, student or not employed }\end{array}$ & $1(7 \%)$ & $2(9 \%)$ & $6(19 \%)$ & $9(13 \%)$ \\
$\quad$ Primary household shopper & $8(53 \%)$ & $19(83 \%)$ & $24(77 \%)$ & $52(75 \%)$ \\
\hline
\end{tabular}

*Non-participants could not be identified because data collected were anonymous within each location; all participated in Maryland; 22 of the 23 in Mississippi, and 20 of 31 in Ohio

${ }^{\dagger}$ Based on $n=30,1$ of the 31 respondents in Ohio did not answer the question

\section{Themes}

Four themes emerged from the analysis: get them early: marketers have got our kids; celebrities are in ads-might not consume what they promote; celebrities featured in silly, stupid, weird, memorable ads; and could celebrities promote healthy products? Detail on each theme follows.

\section{Get Them Early: Marketers Have Got Our Kids}

Participants discussed how children were targeted by marketers from food and beverage companies to make impulsive decisions that might have poor long-term consequences. Such efforts were linked to child(ren)'s requests for specific products, encouragement to consume in general, and promotion of particular brands (including product and/or restaurant brands). Participants pointed out how the developmental stage of a child combined with the exposure to targeted marketing is connecting them to and influencing their consumption of unhealthy food and beverage products. They described marketing through numerous delivery modes, including mail, television (commercials and events), phones (games, apps, internet, social media, and direct marketing texts), and computers. One parent described phone applications that utilized games to directly market to children. The participant stated, "The things that come up on our phone apps when those children are playing with these games are drinks, candy and all those different things that are not good for them, because they're marketing to our children. And they catch our children when they are right there. Then they've got our children." This view of "catch our children" or "bombarding" children was described by other participants as well, where caregivers' described the experience of trying to reduce or eliminate their child(ren)'s exposure to food and beverage marketing, often to no avail. These parents perceived aggressive marketing strategies as aimed at engaging youth early to have life-long customers.

Efforts to reduce exposure were difficult due to the pervasiveness of the marketing targeted to children. One group of participants discussed the exposure to digital marketing, stating that parents might be unaware of some of the marketing that their children are exposed to in the digital world. The participant stated:

As a parent you don't [know] your child is even seeing that and that aspect I have a huge problem with digital marketing as it pertains to younger people because, they're not able to make the best decisions at a certain age.

And another parent described how blocking a child from social media might be ineffective at keeping them from the marketing exposure: "Yeah, you could block them from social media, they're going to see it on someone else's phone." Similarly, another parent described how they previously had not given much thought to the marketing they are exposed to when watching television but now recognized the types of foods and beverages promoted. She said, "it dawned on me...I'm sitting here and I'm watching a cartoon. They're advertising sugary drinks and of course the ramen noodles." Another participant from a different site recalled 
her daughters' awareness of promotional items in fast food meals, stating "they know when figurines from the movie is coming out... [and we] have to go to [fast food restaurant]." Thus, digital and television advertising, along with other promotional strategies, were described as pervasive and influential on children.

\section{Celebrities Are in Ads-Might Not Consume What They Promote}

Celebrities' role in marketing was discussed, with acknowledgement that the celebrity might not be intending to promote unhealthy lifestyles. For example, many participants discussed that it was common for Black celebrities to endorse sugar-sweetened beverages (SSBs). Other participants described how there is a lineage of high-profile celebrities that have represented particular brands, and that in doing certain ads, newer celebrities quickly joined their ranks. In describing the lineage of celebrities endorsing specific products, a participant described what she thought a newer celebrity would think if they were made an offer to endorse that product: "I don't care how much you pay me, I'm doing it because it puts my name on that roster with the rest of them." Participants went on to discuss how endorsing certain national brands, for less healthy products, can elevate their own celebrity status and that celebrity endorsements of certain products have been common across decades:

'Participant B: Endorsing sodas.' Participant A: '[popular singer/songwriter]...get NFL halftime shows and stuff like that. So a lot of the young kids see that. It's being promoted by [SSB brand], so they see that. Just like back in the day, [popular singer/songwriter] had the big [SSB brand] halftime show'.

Thus, participants sought to understand the celebrity's perspective when critiquing their promotion of less healthful products, like SSBs. They articulated how this practice by food marketers is long-standing and results in Black consumers easily connecting a celebrity with certain food/ beverage brands. Participants also added comments about the influence of celebrity endorsements on other, non-food products/services. For example, preferences for specific hairstyles and clothing were described as having been derived from celebrity endorsements or behaviors; participants described children in particular being motivated to make a purchase or change their style.

Some participants questioned the morality of celebrities endorsing unhealthy foods, with one participant challenging them: "If you really thought about your family history and the diseases and stuff that this has caused, would you really be advertising this?" But the same participant went on to say that they were unsure if celebrities had a full understanding of the health impacts of the foods/beverages they endorse:
"But if they really understood what was going on behind the scenes, I don't really think they would." Others similarly asserted that it is unlikely that the celebrity actually consumes the unhealthy foods/beverages they are promoting on a regular basis: “[NBA player], you don't drink this! And if you do, you're probably drink[ing] one can every six months." Thus, consumers described celebrity endorsements as being effective in conveying and fostering (among consumers) a preference for a product but lacking authenticity in the product endorsements.

Participants discussed this disconnect with an appreciation for how one's habits might change over time. They talked about how some celebrities might have a different approach as they age, become wealthier, and have achieved "superstar" status. In speaking of a popular singer endorsing a SSB brand earlier in her career, a participant stated "she didn't know better. Now as a mother of three, I'm pretty sure she would not. I don't care how much money they throw at her, I don't think she'll touch it." Another participant described how a celebrity might endorse a product to "fund their career" but that they might have some regrets later, "And at some point, I think certain people have maybe done something they're not very proud of."

\section{Celebrities Featured in Silly, Stupid, Weird, Memorable Ads}

Participants described what made certain television ads compelling or memorable. After participants shared ads they remembered, they often described the ads as "weird," "stupid conversations," as having "silly" plot lines or "memorable" and "catchy" songs. But in recalling these memorable marketing campaigns, participants appreciated how advertising influences consumer demand such that their desire for a product is increased by effective marketing. Participants often recalled ads that featured a celebrity and stated that the celebrities' presence made it memorable. One participant recalled an ad where a celebrity drinks a SSB when struggling to record a song; he then shatters into pieces revealing he is made of musical circuitry (e.g., speaker components), after which he begins to rap. The participant said, "It was so pointless and stupid, but it just stayed with me forever."

Thus, the combination of visual imagery, albeit unrealistic or silly, and a celebrity results in a memorable commercial. Some participants described how enticing the food/beverages were made to look through imagery on television commercials. One participant described the influence on her behavior, "we're watching television, all of a sudden this [SSB brand] come up. And you won't even be thirsty. But just a look at that [SSB], at that ice, and hear that thing go shh. You get mad going to the refrigerator." One participant shared an opposing view stating that while a jingle/song might be remembered, it does not mean you know or remember much about the company. 
This participant stated that social media usage leads to the repetition of ads (with or without music) to the point that someone viewing a page might feel annoyed at the repetitive nature and/or "filtered out as white noise after a point" because you have seen it so often. The same participant also mentioned that when they see a "sponsored ad" on social media that involves a celebrity, they are less likely to believe that the celebrity's endorsement is sincere.

Other marketing strategies were identified within advertisements as being drivers of consumer demand as well. For example, a participant commented that statements like "limited time" in a commercial could increase the appeal of purchasing a particular item. These "limited" or "special" versions of products were primarily related to beverage flavors or sandwiches at fast food restaurants. Participants also connected this "limited" availability of a product with the role of social media in expanding marketing of food and beverages. One participant recalled the impact of social media on a fast-food chain's new sandwich, stating "they didn't have any advertisements. Social media was doing all the advertising." Here, participants described how demand can be driven by other consumers utilizing social media platforms to promote their approval of the product, and that sometimes celebrities promote products through their own social media posts. These tactics reinforce traditional paid advertising and promotion.

\section{Could Celebrities Promote Healthy Products?}

Participants explicitly recommended potentially having Black celebrities promote healthier products, utilizing the same targeted marketing strategies currently used. There was also interest in ways Black celebrities could promote the health of the community by promoting products and lifestyles that reduce nutrition-related chronic diseases (e.g., diabetes management). One participant more directly described the influence of celebrities:

I kind of feel the celebrity component is very important especially in the African-American community... some pockets of the community tend to look to the celebrities and if they endorse a certain food, they'll be like, okay, if it's good enough for them, then it's good enough for me.

Some participants expressed that the mix of marketing was "getting better," but other participants talked about continued efforts to target Black communities with unhealthy products. "Day and night between the way they target us for sugary drinks and how they use our [Black] artists to promote certain foods." One participant described how the Black community is ignored for healthful products, saying:
I feel like we're targeted for a lot of stuff. And I'm not sure why we are targeted for a lot of stuff when it comes to food and beauty and clothing and shoes, but when it comes to health, medicine, mental health, it falls flat.

Similarly, a participant, who is a healthcare provider, stated they would like to see commercials for diabetes management for the community: "more of that, instead of Black folks promoting [sneaker brand] and sugary drinks and [fast food chain]."

There were some mentions of celebrities promoting healthy products in the past, such as a famous NBA player promoting a low sugar, whole wheat cereal that influenced them to purchase and consume the healthier cereal:

$\ldots$ if there are rappers or something like that in a commercial eating healthy, they would do it. They would follow though because [NBA player] did the [Cereal brand] commercial. We was eating it back then. We was eating it the same.

Participants also pointed out that celebrities are more commonly promoting healthy foods in relation to lifestyles they adhere to. Their promotion may or may not be related to specific business relationships. One group referenced a few celebrities:

'Participant A: '[Talk show host] got that healthy too.'

... Participant B: 'You know she advertised her food,

whatever it is she be doing, like I said.' Participant C:

'She's the spokesperson for [weight loss company].

She's a national spokesperson.'

Here participants connected a celebrity's healthy lifestyle to relationships with food and beverage wellness companies. The same group described celebrities promoting their vegan lifestyle, which was not connected to business relationships with any specific food and beverage companies.

Participants described the need for more marketing of healthy foods in Black communities. Moderators inquired about a potential approach, one specifically asking, "Who do you think should lead the effort to create a broader variety of healthy foods and beverages in the community?". Participants replied by naming certain celebrities or generally endorsing the use of celebrities to promote healthier foods for the Black community. There was support for utilizing the influence of celebrities to promote a healthier mix of foods and beverages. One participant stated: “... I think that's an absolutely great idea when she said that we should buy into getting the celebrities to go on television and start advertising more in the Black community things that are healthy to eat."

One participant described wanting to see a switch in the advertising emphasis to see if it had an effect: "I would love 
to see them stop all the advertising of bad foods and then advertise fruits and see if it get the same effect." Another stated: "If they really marketed a bunch of fruits and vegetables and different ways to eat healthy, I wonder if people would try it just as if they was going to try [NBA player]'s sugary drink." Other participants saw the opportunity to take advantage of how children admire celebrities. One participant proposed, "If we could get a role model that these kids love.... and have them come on and say, 'Hey kids! Drink water. Don't drink [SSB brand].' I bet you the kids would go, 'Wow. He's drinking water. Maybe I'll drink water."' Another participant challenged celebrities to take advantage of their position and to "build for the future" by promoting items with less sugar and "healthier" ingredients. Several participants wondered how feasible it would be to get celebrities to adjust endorsements to healthier items. One asked, "How do you get to those people to say, 'Change your message.' I mean, how do you get to them?" and another responded, "I don't think you can get to them." While there was uncertainty on the probability of this change occurring, there was clear support for the idea that this strategy should be assessed for impact on Black community health.

\section{Discussion}

This study adds to the still very limited evidence about Black consumer perceptions and opinions about ethnically targeted marketing and its implications for Black community health. It also identifies celebrity endorsements as a specific action issue that these Black community members believed worthy of pursuing. The four salient themes that emerged from our participants were similar, regardless of location. One theme focused on concerns about marketing to children - get them early, marketers have got our kids; two related directly to celebrities' roles in FBHFS marketing-celebrities are in ads-might not consume what they promote; and celebrities featured in silly, stupid, weird, memorable ads. The othercould celebrities promote healthy products? related to potential celebrity roles in marketing healthy foods and beverages and reflected a cross-cutting concern about potential adverse influences of targeted FBHFS marketing to Black consumers.

Despite the fact that only one scenario mentioned youth, participants recognized that advertising exposures lead youth to purchase requests for FBHFS. Their perceptions were consistent with research which describes how social media engagement can be especially influential for youth given their developmental susceptibility and emphasis on unique peer-to-peer communication [12, 15, 39]. Participants also emphasized youth affinity for celebrities when discussing celebrities' promotional influence. The concerns about youth-oriented marketing are consistent with evidence that Black caregivers are concerned about child obesity and feel a strong responsibility for their children's eating habits [40]. When made aware of the various tactics used, Black parents indicate specific concerns about food marketing to their children and interest in supporting certain types of policy solutions [34, 36]. A Rudd Center report [36], which was based on ethnicity-specific focus groups with parents about food marketing to children, included limited detail on specific findings from the Black parent group but did report that parents were dissatisfied with some aspects of Black targeted advertisements, i.e., that they were associated primarily with unhealthy foods and included only Black rather than a more diverse set of actors.

The high salience to study participants of issues related to celebrities was also noteworthy given that only 4 of the 19 scenarios featured celebrities. Nonetheless, celebrity endorsements, including the use of licensed brand characters (such as those used with breakfast cereals) or fictional or cartoon characters popular with children, have been prominent public health issues for some time [6]. Participants identified potential roles of celebrities across all four elements of a "marketing funnel"- a concept commonly used to describe the process of moving a consumer from awareness of the product, cascading to developing more specific interest in and desire for the product and purchase intention culminating in a purchase [41]. The strategic use of Black celebrities provides an ethnic cue which increases the salience of the identity and evokes more identity-consistent and favorable responses from Black youth [42]. Specifically, celebrities build awareness by lending their stature and visibility to a branded product and create interest by the way they talk about or engage with it and link to their own lifestyle. This creates desire among those who idolize and wish to be like them, and product purchase fulfills that desire. This characterization reflects how marketers strategically use celebrities in diverse ways to persuade consumers and facilitate purchase. Participants recognized how celebrities are used to target Black consumers through diverse media platforms in both paid advertising and more subtle indirect strategies and tactics.

Participant comments about the potential for Black celebrities to market healthy foods were optimistic. They reflected perceived commonality with celebrities around Black identity-a sense that Black celebrities should be sensitive to the health issues of Black communities. Food and beverage companies leverage most celebrity marketing expenditures to promote FBHFS [43]. However, participants thought that celebrities-especially once reputations were establishedcould be convinced to reconsider FBHFS endorsements on principle, by using the argument that they are harming their own communities. Although aware that endorsements are lucrative, participants but did not discuss whether loss of the large sums of money would be a deterrent. They did voice 
skepticism as to whether celebrities would be as effective when endorsing healthy compared to less healthy products. There is precedent for Black celebrity involvement in marketing campaigns for fruits and vegetables and water [44], although some celebrities who market fruits and vegetables pro bono also have concurrent contracts to market FBHFS [45].

Strengths of our study include the methodology incorporating multiple types of marketing practices, including examples of television, music lyrics, and social media advertising, as well as digital marketing and a two-session design that allowed for awareness raising, an opportunity for reflection during the interim and continued discussion. The study objectives were met despite technical issues that limited the number of audio files obtained. However, the study sample was a small, convenience sample not representative of the respective geographic areas, and both recruitment and participation were impacted by holidays and competing community events. In addition, the advent of the COVID-19 pandemic in early 2021 prevented two of the sites from convening their in-person community meetings. Timely virtual options were not feasible. Nevertheless, data were obtained from all three sites and from both age groups, and provided a rich dialogue reflecting perspectives, concerns, and insights of diverse Black community members.

\section{Conclusion}

Youth-oriented efforts to address ethnically targeted marketing, as suggested by our findings, may be strategic in their focus. Caregivers are household food providers and role models and shape youth eating patterns, media use, and nutrition literacy in other ways [46-48]. In addition, the adults in our study may have had difficulty seeing how they are personally affected by marketing, while seeing that others (youth) are affected-a phenomenon termed a "third person effect" in communications research [49]. The prior consumer sensitization study, which examined and identified certain concerns about fairness and social justice among Black adults, also identified tolerant attitudes related to targeted marketing, e.g., concepts of personal choice and acceptance as normal business practices [35]. At the same time, youth-led advocacy related to healthy food promotion has shown promising results [50-52]. These findings raise the question of whether youth advocacy, with parent involvement, could convince more Black celebrities to promote healthy foods.

Our findings, including the observation that latent concerns can be elicited when awareness is increased by exposure to examples of marketing tactics, align with previous reports that probed specifically for concerns related to youth marketing $[34,36,40]$. However, these findings also indicate a need for further qualitative exploration to obtain a deep and nuanced understanding of how Black consumers view ethnically targeted FBHFS marketing generally and might be convinced to support policies to reduce it.

Acknowledgements We acknowledge the colleagues who led the recruitment and implementation of this study in their communities: Sandra Chapelle, Sydney Daigle, Linda McGee, and other members of the local study teams as well as Kimberly Alexander, Sara Ribacove, and Dr. Anne Palmer for their assistance with project implementation. We are also grateful to the community members who participated in this study and shared their perspectives and experiences with us.

Author Contribution Shiriki Kumanyika, Katie Isselmann DiSantis, Sonya Grier, and Tiffany M. Eaton contributed to the design of the study protocol and materials. Tiffany M. Eaton and Kenna Yadeta coded the focus group transcripts and collaborated with Katie Isselmann DiSantis and Shiriki Kumanyika on the thematic analyses. All authors collaborated in the development, drafting, and editing of the manuscript.

Funding This research was supported by a grant from the Bloomberg American Health Initiative at the Johns Hopkins Bloomberg School of Public Health and NIH, NIDDK Grant P30DK092950.

Availability of Data and Material Not applicable.

Code Availability Not applicable.

\section{Declarations}

Ethics Approval This study was conducted by the Council on Black Health, hosted at Drexel University, and was approved as exempt by the Drexel University Institutional Review Board (IRB\#: 1907007272).

Consent to Participate Not applicable.

Consent for Publication Not applicable.

Conflict of Interest The authors declare no competing interests.

Disclaimer The authors take sole responsibility for the article content.

\section{References}

1. Ogden CL, et al. Trends in obesity prevalence by race and hispanic origin-1999-2000 to 2017-2018. JAMA. 2020;324:1208-10. https://doi.org/10.1001/jama.2020.14590.

2. Hales CM, Carroll MD, Fryar CD, Ogden CL. Prevalence of obesity and severe obesity among adults: United States, 2017-2018. NCHS Data Brief. 2020;(360):1-8. https://www.cdc.gov/nchs/ data/databriefs/db360-h.pdf.

3. Dietary Guidelines Advisory Committee. Scientific report of the 2020 dietary guidelines advisory committee: advisory report to the secretary of agriculture and the secretary of health and human services. 2020. Washington, DC: U.S. Department of Agriculture, Agricultural Research Service.

4. Institute of Medicine. Institute of medicine. 2012. Accelerating progress in obesity prevention: solving the weight of the nation. 
Washington, DC: The national Academies Press. Adv Nutr. 2012;3:708-9. https://doi.org/10.3945/an.112.002733.

5. Kraak VI, et al. Progress achieved in restricting the marketing of high-fat, sugary and salty food and beverage products to children. Bull World Health Organ. 2016;94:540-8. https://doi.org/10.2471/ blt.15.158667.

6. Institute of Medicine. Food marketing to children and youth. Threat or opportunity? Washington, DC: National Academies Press; 2006.

7. Powell LM, Harris JL, Fox T. Food marketing expenditures aimed at youth: putting the numbers in context. Am J Prev Med. 2013;45:453-61. https://doi.org/10.1016/j.amepre.2013.06.003.

8. Harris JL, et al. A crisis in the marketplace: How food marketing contributes to childhood obesity and what can be done. Annu Rev Public Health. 2009;30:211-25. https://doi.org/10.1146/annurev. publhealth.031308.100304.

9. Federal Trade Commission. A review of food marketing to children and adolescents. Follow-up report. Washington, DC. 2012. https://www.ftc.gov/sites/default/files/documents/reports/reviewfood-marketing-children-and-adolescents-follow-report/12122 1 foodmarketingreport.pdf. Accessed 18 August 2021.

10. Cervi MM, et al. Susceptibility to food advertisements and sugarsweetened beverage intake in non-hispanic black and non-hispanic white adolescents. J Community Health. 2017;42:748-56. https:// doi.org/10.1007/s10900-016-0313-4.

11. Fleming-Milici F, Harris JL. Adolescents engagement with unhealthy food and beverage brands on social media. Appetite. 2020;146:104501. https://doi.org/10.1016/j.appet.2019.104501.

12. Montgomery KC, Chester J. Interactive food and beverage marketing: targeting adolescents in the digital age. J Adolesc Health. 2009;45:S18-29. https://doi.org/10.1016/j.jadohealth.2009.04. 006.

13 Murphy G, et al. See, like, share, remember: adolescents' responses to unhealthy-, healthy- and non-food advertising in social media. Int J Environ Res Public Health. 2020;17(7):2181. https://doi.org/10.3390/ijerph17072181.

14. Potvin Kent M, et al. Children and adolescents' exposure to food and beverage marketing in social media apps. Pediatr Obes. 2019;14:e12508. https://doi.org/10.1111/ijpo.12508.

15 Montgomery $\mathrm{KC}$, et al. The new threat of digital marketing. Pediatr Clin North Am. 2012;59:659-75. viii.https://doi.org/10.1016/j. pcl.2012.03.022.

16. Powell LM, Wada R, Kumanyika SK. Racial/ethnic and income disparities in child and adolescent exposure to food and beverage television ads across the U.S. Media markets. Health Place. 2014;29:124-31. https://doi.org/10.1016/j.healthplace.2014.06. 006.

17. Adeigbe RT, et al. Food and beverage marketing to latinos: a systematic literature review. Health Educ Behav. 2015;42:569-82. https://doi.org/10.1177/1090198114557122.

18. Nguyen $\mathrm{KH}$, et al. Transferring racial/ethnic marketing strategies from tobacco to food corporations: Philip Morris and Kraft General Foods. Am J Public Health. 2020;110:329-36. https://doi.org/ 10.2105/ajph.2019.305482.

19. Backholer K, et al. Differential exposure to, and potential impact of, unhealthy advertising to children by socio-economic and ethnic groups: a systematic review of the evidence. Obes Rev. 2021;22:e13144. https://doi.org/10.1111/obr.13144.

20. Thai CL, et al. Perceptions of food advertising and association with consumption of energy-dense nutrient-poor foods among adolescents in the United States: results from a national survey. $\mathbf{J}$ Health Commun. 2017;22:638-46. https://doi.org/10.1080/10810 730.2017.1339145.

21. Fleming-Milici F, Harris JL. Television food advertising viewed by preschoolers, children and adolescents: contributors to differences in exposure for black and white youth in the united states.
Pediatr Obes. 2018;13:103-10. https://doi.org/10.1111/ijpo. 12203.

22. Grier SA, Kumanyika SK. The context for choice: health implications of targeted food and beverage marketing to african americans. Am J Public Health. 2008;98:1616-29. https://doi.org/10. 2105/AJPH.2007.115626.

23. Grier SA, Kumanyika S. Targeted marketing and public health. Annu Rev Public Health. 2010;31:349-69. https://doi.org/10. 1146/annurev.publhealth.012809.103607.

24. Williams JD, et al. The role of food culture and marketing activity in health disparities. Prev Med. 2012;55:382-6. https://doi.org/10. 1016/j.ypmed.2011.12.021.

25. Harris JL, Frazier W, Kumanyika S, Ramirez AG. Increasing disparities in unhealthy food advertising targeted to Hispanic and Black youth. January 2019. Available at: http://uconnruddcenter. org/files/Pdfs/TargetedMarketingReport2019.pdf. Accessed 18 August 2021.

26. Whalen R, et al. Children's exposure to food advertising: the impact of statutory restrictions. Health Promot Int. 2019;34:22735. https://doi.org/10.1093/heapro/dax044.

27. Boyland E, et al. Digital food marketing to young people: a substantial public health challenge. Ann Nutr Metab. 2020;76:6-9. https://doi.org/10.1159/000506413.

28. Kumanyika SK. A question of competing rights, priorities, and principles: a postscript to the Robert Wood Johnson Foundation Symposium on the ethics of childhood obesity policy. Prev Chronic Dis. 2011;8:A97.

29. Commercial speech. Cornell Law Review Legal Information Institute. https://ww.wlaw.cornell.edu/constitution-conan/amend ment-1/commercial-speech. Accessed 14 May 142021.

30. Bragg MA, et al. Evaluating the influence of racially targeted food and beverage advertisements on black and white adolescents' perceptions and preferences. Appetite. 2019;140:41-9. https://doi. org/10.1016/j.appet.2019.05.001.

31. Harris J, et al. A qualitative assessment of us black and latino adolescents' attitudes about targeted marketing of unhealthy food and beverages. J Child Media. 2019;13:295-316. https://doi.org/ 10.1080/17482798.2019.1604394.

32. Wootan MG, et al. How do nutrition guidelines compare for industry to market food and beverage products to children? World health organization nutrient profile standards versus the us children's food and beverage advertising initiative. Child Obes. 2019;15:194-9. https://doi.org/10.1089/chi.2018.0256.

33. Schermbeck RM, Powell LM. Nutrition recommendations and the children's food and beverage advertising initiative's 2014 approved food and beverage product list. Prev Chronic Dis. 2015;12:E53. https://doi.org/10.5888/pcd12.140472.

34 Fleming-Milici F, Harris JL, Liu S. Race, ethnicity, and other factors predicting U.S. parents' support for policies to reduce food and beverage marketing to children and adolescents. Health Equity. 2018;2:288-95. https://doi.org/10.1089/heq.2018.0048.

35. DiSantis KI, et al. Sensitizing black adult and youth consumers to targeted food marketing tactics in their environments. Int J Environ Res Public Health. 2017;14(11):1316. https://doi.org/10.3390/ ijerph14111316.

36. Ustjanauskas A, Eckman, B, Harris, JL, Goren A, Schwartz, M, Brownell KD. Rudd report: focus groups with parents. What do they think about food marketing. New Haven: Yale University; 2010. http://www.Uconnruddcenter.Org/files/pdfs/ruddr eport_focusgroupsparents_5_10.Pdf. Accessed 18 August 2021.

37. Kamberelis G, Dimitriadis G. Focus groups: contingent articulations of pedagogy, politics and inquiry. In: Denzin NK, Lincolc YS, editors. The SAGE handbook of qualitative research. Los Angeles: Sage; 2011. p. 545-61.

38. Kotler P, Armstrong G. Principles of marketing. 12th ed. New Jersey: Prentice Hall; 2008. 
39. Harris JL, Brownell KD, Bargh JA. The food marketing defense model: integrating psychological research to protect youth and inform public policy. Soc Issues Policy Rev. 2009;3:211-71. https://doi.org/10.1111/j.1751-2409.2009.01015.x.

40. Johnson-Askew WL, et al. Attitudes of african american advocates toward childhood obesity. Ethn Dis. 2011;21:268-73.

41. Court D, Elzinga D, Mulder S, Vetvik OJ. The consumer decision journey. The McKinsey Quarterly; 2009. Available at: https:// www.Mckinsey.Com/business-functions/marketing-and-sales/ our-insights/the-consumer-decision-journey. Accessed 18 August 2021.

42. Grier SA, Berkeley Media Studies Group and National Public Health Law Network. African American \& Hispanic youth vulnerability to target marketing: implications for understanding the effects of digital marketing. Memo prepared for the second nplan/bmsg meeting on digital media and marketing to children. 2009. http://digitalads.org/how-youre-targeted/publications/ african-american-hispanic-youth-vulnerability-target-marketing. Accessed 18 August 2021.

43. Zhou M, et al. Mapping the celebrity endorsement of branded food and beverage products and marketing campaigns in the united states, 1990-2017. Int J Environ Res Public Health. 2019;16(19):3743. https://doi.org/10.3390/ijerph16193743.

44. Englund TR, et al. Evaluation of integrated marketing communication strategies used for the fruits \& veggies campaign in california and virginia. Prev Med Rep. 2020;18:101062. https://doi. org/10.1016/j.pmedr.2020.101062.

45. Kraak VI, Zhou M, Duffey KJ, Williams JD. A profile of celebrities involved in the partnership for a healtheir america's fruits and veggies (fnv) campaign: 2015-2016 sellout for fnb 2017. Department of Human Nutrition, Foods, and Exercise, College of Agriculture and Life Sciences, Virginia Tech: Blacksburg. https:// vtechworks.lib.vt.edu/bitstream/handle/10919/102773/VT\%20Stu
dy\%204_FNV\%20Celebrity\%20Profile\%20Report_01312017_ FINAL.pdfsequence $=2 \&$ is Allowed $=\mathrm{y}$.

46. Grier SA, et al. Fast-food marketing and children's fast-food consumption: exploring parents' influences in an ethnically diverse sample. J Public Policy Mark. 2007;26:221-35. https://doi.org/ 10.1509/jppm.26.2.221.

47. Parks EP, et al. Perspectives on stress, parenting, and children's obesity-related behaviors in black families. Health Educ Behav. 2016;43:632-40. https://doi.org/10.1177/1090198115620418.

48. Parks EP, et al. Influence of stress in parents on child obesity and related behaviors. Pediatrics. 2012;130:e1096-104. https://doi. org/10.1542/peds.2012-0895.

49. Davison WP. The third-person effect in communication. Public Opin Q. 1983;47:1-15.

50. Frerichs L, et al. Process and outcomes from a youth-led campaign to address healthy eating in an urban high school. J Prim Prev. 2015;36:433-8. https://doi.org/10.1007/s10935-015-0409-0.

51. Leung MM, et al. Voices through cameras: using photovoice to explore food justice issues with minority youth in east harlem, new york. Health Promot Pract. 2017;18:211-20. https://doi.org/ $10.1177 / 1524839916678404$.

52 Schillinger D, Tran J, Fine S. Do low income youth of color see "the bigger picture" when discussing type 2 diabetes: a qualitative evaluation of a public health literacy campaign. Int J Environ Res Public Health. 2018;15(5):840. https://doi.org/10.3390/ijerph1505 0840.

Publisher's note Springer Nature remains neutral with regard to jurisdictional claims in published maps and institutional affiliations. 